\title{
Digital ulcers secondary to Sneddon's syndrome successfully treated with Bosentan: not only useful in Systemic Sclerosis
}

\author{
Tommaso Barnini • Elena Silvestri • \\ Giacomo Emmi • Mario Milco D'Elios • \\ Lorenzo Emmi
}

Received: 20 September 2012/ Accepted: 24 September 2012/Published online: 9 October 2012

(C) Springer-Verlag Italia 2012

Sir,

Sneddon's Syndrome is a rare and poorly understood syndrome characterized by the association of ischemic cerebrovascular events and livedo racemosa [1]. We report a case of a 35-year-old woman with Sneddon's Syndrome and digital ulcers successfully treated with Bosentan.

Patient was admitted to our center with pain and trophic ulcers in both hands and feet, Raynaud's phenomenon, livedo racemosa on the extremities, headache and memory loss. Her past medical history was notable for Cushing syndrome treated with total hypophysectomy and bilateral adrenalectomy, a history of recurrent miscarriage and hypertension under treatment. Physical examination was normal except for the skin lesions. The search for antiphospholipid antibodies [anticardiolipin (aCL), anti-beta2GPI and lupus anticoagulant (LA)], antinuclear autoantibodies (ANA), anti-extractable nuclear antigens (ENA) and antidouble-stranded DNA (anti-dsDNA) was negative. Cerebral MRI showed multiple subcortical and periventricular lesions suggestive of previous ischemic events. In the light of these findings, a carotid ultrasonography and an echocardiography were performed to rule out micro embolism. Echocardiography showed a partial thickening of the mitral valve. Livedo racemosa was confirmed by a skin biopsy showing a light leukocyte infiltrate and capillary dilatation. The diagnosis of Sneddon's Syndrome was made. Due to the lack of ulcer's healing with conventional therapies we decided to replace iloprost pulse therapy with Bosentan

T. Barnini $(\bowtie) \cdot$ E. Silvestri · G. Emmi ·

M. M. D'Elios · L. Emmi

SOD Patologia Medica, Center for Autoimmune Diseases,

Lupus Clinic, DAI Biomedicina, AOU Careggi,

Largo Giovanni Brambilla 3, 50134 Florence, Italy

e-mail: tommasobarnini@gmail.com treatment. Bosentan is a dual endothelin-1 (ET-1) receptor antagonist with affinity for both ET-1 A and ET-1 B receptors. ET-1 is one of the most powerful vasoconstrictor agents [2], able to induce fibrosis and cellular proliferation.
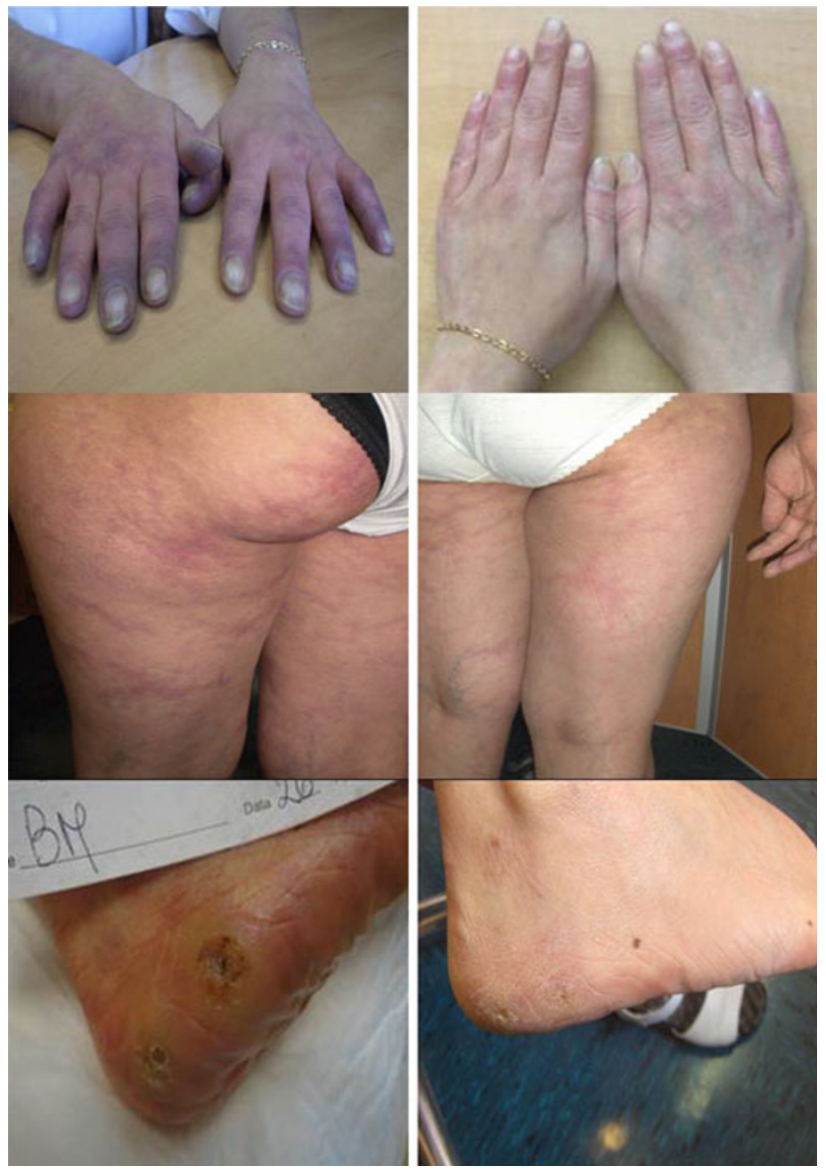

Fig. 1 Patient's livedo and trophic ulcers before (left) and after (right) Bosentan treatment 
These effects were mediated by the ET-1 binding on both receptors in vascular smooth muscle cells. Bosentan has been approved by the European Medicines Agency (EMA) for the prevention of new digital ulcer formation in Systemic Sclerosis (SS) [3]. A recent study on patients with SS demonstrated a $30 \%$ reduction of new digital ulcers during treatment with Bosentan but no healing of active ulcers was observed [4].

In our patient, a few weeks after the start of Bosentan administration, ulcers rapidly healed, with an important reduction of pain and livedo (Fig. 1). Clinical conditions remained good and no new ulcers developed over the next 6 months.

To our knowledge, this is the first case of Sneddon's Syndrome treated with Bosentan; in contrast to that reported in SS, Bosentan led to a complete healing of preexisting cutaneous ulcers.

The success of this anti-endothelial proliferative drug supports the hypothesis [5] that an endothelial proliferation could be the main pathophysiological mechanism of this obliterative vasculopathy.
Conflict of interest The authors declare no conflict of interest.

\section{References}

1. Francès C, Piette JC (2000) The mystery of Sneddon syndrome: relationship with antiphospholipid syndrome and systemic lupus erythematosus. J Autoimmun 15:139-143

2. Wigley FM (2009) Vascular disease in scleroderma. Clin Rev Allergy Immunol 36:150-175

3. European Medicines Agency (2009) Tracleer (bosentan) $62.5 \mathrm{mg}$ film-coated tablets: summary of product characteristics. http://www. ema.europa.eu/docs/en_GB/document_library/EPAR_-_Summary_ for_the_public/human/000401/WC500041455.pdf

4. Matucci-Cerinic M, Denton CP, Furst DE et al (2011) Bosentan treatment of digital ulcers related to systemic sclerosis: results from the RAPIDS-2 randomised, double-blind, placebo-controlled trial. Ann Rheum Dis 70:2-38

5. Sepp N, Zelger B, Schuler G et al (1995) Sneddon's syndrome: an inflammatory disorder of small arteries followed by smooth muscle proliferation. Immunohistochemical and ultrastructural evidence. Am J Surg Pathol 19:448-453 